HARDY-RAMANUJAN JOURNAL 34 \& 35 (2013), 47-52

\title{
MATHEMATICAL REMINISCENCES: HOW TO KEEP THE POT BOILING
}

\author{
K. RAMACHANDRA
}

Edited specially for this volume from a handwritten manuscript of Ramachandra by K. Srinivas.

\section{INTRODUCTION}

Analytic Number Theory is no doubt the most interesting subject. It deals with the application of Analysis, both real and complex, to the study of Numbers. It includes Primes, Transcendental Numbers, Diophantine equations and other questions on $1,2,3,4,5, \ldots$. Study of the Riemann zeta-function $(\zeta(s))$ is intimately connected with that of primes. As an elderly ${ }^{1}$ gentleman, who has worked in Analytic Number Theory for more than forty five years, I am concerned with the following problem: How can a beginner contribute a paper and how to be a professional mathematician sustaining research activity throughout? There is no general solution. One way is to study the papers by G. H. Hardy, J. E. Littlewood, I. M. Vinogradov, P. Erdös, S. S. Pillai, S. D. Chowla, R. P. Bambah, K. Ramachandra, R. Balasubramanian, T. N. Shorey and others. Unless there is a strong group of people interested in this area at a concentrated place or state, the school which S. Ramanujan would have liked will be unheard of in the days to come. It is already so and we are disappointed at this.

Below we list some problems which are not easy but likely to be solved in not too distant a future. They may be difficult. These are not the only problems to be solved. But students can get an idea of what problems are not likely to be too difficult by talking to experts. Now I consider the problem How to keep the pot boiling. I list a few problems which may be very difficult, but probably there is some chance of being solved. A great monumental book to be written in golden letters is the book by G. H. Hardy [2], which every aspiring researcher must read.

\footnotetext{
${ }^{1}$ Ramachandra mentions his age as 71 at the time of writing this article; since he was born in the 1933, we can presume that this may have been written in 2004 .
} 


\section{Problems.}

1. I begin with a problem by M.Jutila, myself and T. N. Shorey ([7], [8]). Let $k \geq 1000$ be any fixed integer. Let $n_{1}, n_{2}, n_{3}, .$. be the sequence of all positive integers all of whose prime factors are $\leq k$. It is trivial to prove that the maximum gap is finite and $\leq k$ !. In a series of papers (written individually and jointly) we proved that

$$
\text { (maximum gap) }\left\{\mathrm{k}(\log \mathrm{k})^{-1} \frac{\log \log \log \mathrm{k}}{\log \log \mathrm{k}}\right\}^{-1}
$$

is bounded above by a constant $C>0$. To compute admissible constant $C$ is not easy and was done by Stephen Astels in his master's thesis in 1995. The title of the thesis is On integers with a large prime factor. In fact, he worked out an explicit version of the result in [7]. The problem is to prove that $C$ can be replaced by any $\epsilon>0$ and all $k \geq k_{0}(\epsilon)$.

2. Let $a$ and $b$ be non-zero complex numbers such that $a / b$ is irrational. It is not hard to prove that $\exp (a z)$ and $\exp (b z)$ are algebraically independent. Consider the set $V_{a}$ of complex numbers $\alpha$ such that $\exp (a \alpha)$ is algebraic. C. L. Siegel and T. Schneider proved that as a vector space over rationals $\operatorname{dim}\left(V_{a} \cap V_{b}\right) \leq 2$ (rediscovery by S. Lang and myself independently of each other). Using this it was possible to deduce as a corollary $([4])$ the following. Let $t$ be any fixed transcendental number. Consider the number $f(N)$ of algebraic numbers in the finite set

$$
2^{t}, 2^{t^{2}}, 2^{t^{3}}, \ldots, 2^{t^{N}}
$$

Then for all $\epsilon>0$ and all $N \geq N_{0}(\epsilon)$, we have

$$
f(N) \leq \sqrt{(2 N)}(1+\epsilon) .
$$

Improve the constant 2.

3. The famous Catalan's conjecture (now a theorem of P. Mihăilescu (see [9]))says that the numbers $8\left(=2^{3}\right)$ and $9\left(=3^{2}\right)$ are the only consecutive numbers which are powers. R. Tijdeman proved that if $n \geq N_{0}$ (an effective constant) then both $n$ and $n+1$ cannot be powers. His proof depended very much on the deep work of $\mathrm{A}$. Baker on linear forms in the logarithms of algebraic numbers. Things like $n, n+2$ cannot both be powers if $N \geq N_{0}^{\prime}$ are very much open. S. S. Pillai had proposed the following

Conjecture. Let $a, b, c$ be fixed positive integers. Then the equation

$$
a x^{m}-b y^{n}=c(m>1, n>1, m n \geq 6)
$$

has only finitely many quadruplets of positive integers $(x, y, m, n)$.

Of course $x^{2}-61 y^{2}=1$ has infinitely many solutions in positive integers $x, y$. 
4. Let $s_{1}, s_{2}, \ldots$ be the sequence of integers (in the increasing order) represented by the quadratic form $x^{2}+y^{2}$. Then $s_{n+1}-s_{n}=O\left(s_{n}^{\frac{1}{4}}\right)$ is trivial since $\max _{x}\left|x^{2}-n\right|=$ $O\left(n^{\frac{1}{2}}\right)$. S. D. Chowla and R. P. Bambah have proposed the problem of proving $s_{n+1}-s_{n}=o\left(s_{n}^{\frac{1}{4}}\right)$.

5. It is well known that $\sin \frac{\pi}{9}$ is an algebraic number of degree 3 but not expressible in terms of real radicals. In his fundamental paper (see [10]) S. Ramanujan has expressed $j(\alpha)$ ( $\alpha$ imaginary quadratic) and similar values of related modular functions in terms of real radicals for some special numbers $\alpha$. By the theory of complex multiplication $j\left(\frac{a+b \sqrt{ } d}{c}\right)(a, b, c, d$ integers with $d<0, b / c>0)$ are all algebraic numbers. Characterise those $(a, b, c, d)$ for which $j\left(\frac{a+b \sqrt{ } d}{c}\right)$ can be expressed in terms of real radicals.

6. On Riemann hypothesis we have $p_{n+1}-p_{n} \ll p_{n}^{\frac{1}{2}} \log p_{n}$ (where $p_{n}$ is the $n^{t h}$ prime), but we have no way of proving $p_{n+1}-p_{n} \ll p_{n}^{\frac{1}{2}}$. I defined for $(n \geq 1, \alpha>0), P(n, \alpha)$ to be the maximum prime factor of $n+1, n+2, \ldots,\left[n+n^{\alpha}\right]$. I proved that for some $\alpha<\frac{1}{2}$ we have $P(n, \alpha) \gg n^{\frac{1}{2}+\frac{1}{13}}$ (without any hypothesis.) This question was taken up by many mathematicians and they have improved this. There was a considerable progress if $\alpha=\frac{1}{2}+\epsilon(\epsilon>0$ any constant). The latest is by D. R. Heath-Brown and Ming-Chit-Liu who proved $P(n, \alpha) \gg n^{\frac{25}{26}}$ if $\alpha>\frac{1}{2}$. Try to work around this problem (prove $P\left(n, \frac{1}{2}\right) \gg n^{1-\epsilon}$ on $\mathrm{RH}$ ).

7. Let $a_{1}, a_{2}, \ldots$ be any sequence of complex numbers with $\sum_{n \leq x} a_{n}=O(1)$. Let $f(s)=\sum_{n=1}^{\infty} \frac{a_{n}}{n^{s}}, \sigma>0$.

(a) Prove that $\zeta(s)+f(s)$ has infinitely many zeros in $\sigma \geq \frac{1}{2}$.

Remark. R. Balasubramanian and myself have proved that there are $\gg_{\delta} T \log T$ zeros in $\left(\sigma \geq \frac{1}{2}-\delta, T \leq t \leq 2 T\right)$ for any fixed $\delta>0$.

(b) Prove that

$$
\int_{T}^{2 T}|\zeta(s)+f(s)|_{\sigma=\frac{1}{2}} d t \gg \int_{T}^{2 T}|\zeta(s)|_{\sigma=\frac{1}{2}} d t
$$

Remark. This was proved with $\sigma=\frac{1}{2}-\delta$ by R. Balasubramanian and myself.

(c) Prove that the number of zeros of $\zeta(s)+f(s)$ in $\left(\sigma \geq \frac{1}{2}-\delta, T \leq t \leq T+T^{1-\epsilon}\right)$ is $\gg T^{1-\epsilon} \log T$ for every fixed $\delta>0$ and $\epsilon>0$.

8. Develop a general theory of zeros of Dirichlet series (and more generally of generalised Dirichlet series). R. Balasubramanian and myself wrote 19 papers on this topic (sometimes jointly and sometimes individually; one of them is a joint paper with A. Sankaranarayanan). We considered Dirichlet series $F(s)=$ 
$\sum_{n=1}^{\infty} b_{n} n^{-s}$ (and more generally generalised Dirichlet series) with $\sum_{x \leq n \leq 2 x}\left|b_{n}^{2}\right| \gg_{\epsilon}$ $x^{1-\epsilon}$ for every $\epsilon>0$. We assumed that $F(s)$ can be continued analytically in $\left(\sigma \geq \frac{1}{2}-\delta, t \geq t_{0}\right)$ and there $|F(s)|<t^{A}$ where $A>0$ is a constant. We proved that the number of zeros of $F(s)$ in $\left(\sigma \geq \frac{1}{2}-\frac{\delta}{2}, T \leq t \leq 2 T\right)$ is $\gg T^{1-\epsilon}$ for every $\epsilon>0$. Improve this to $\gg T \log T$. Various results have been proved in 19 papers.

9. Assuming the Riemann Hypothesis, E. C. Titchmarsh has proved that

$$
\left|\sum_{n \leq x} \mu(n)\right| \leq x^{\frac{1}{2}+\theta} \text { where } \theta=\frac{\mathrm{c}}{\log \log x}(\mathrm{c}>0) .
$$

Recently H. L. Montgomery and H. Maier have replaced $\frac{c}{\log \log x}$ by $c(\log x)^{-\frac{22}{61}}$.

10. A. Selberg has proved that

$$
\left|\zeta\left(\frac{1}{2}+i t\right)\right| \leq t^{\phi}, \text { where } \phi=\frac{\mathrm{c}}{\log \log \mathrm{t}}(\mathrm{c}>0, \mathrm{t} \geq 1000) .
$$

Problem. Improve $\log \log t$ to $(\log \mathrm{t})^{\lambda}$ for some $\lambda>0$.

11. Myself and A. Sankaranarayanan have proved that

$$
\min _{T \leq t \leq 2 T} \max _{\sigma}|\zeta(s)|^{-1} \leq \exp \left(c(\log \log T)^{2}\right) .
$$

Improve this.

12. On Riemann Hypothesis, A. Selberg has proved that

$$
\gamma_{n+1}-\gamma_{n} \ll\left(\log \log \gamma_{n}\right)^{-1}
$$

where $\gamma_{n}$ is the ordinate of the $n^{\text {th }}$ zero of the Riemann zeta function. Improve this. Unconditional results of the type

$$
\gamma_{n+1}-\gamma_{n} \ll \gamma_{n}^{\alpha} \log \gamma_{n}{ }^{\beta}
$$

are available as follows:

R. Balasubramanian: $\alpha=1 / 6+\epsilon, \beta=0$, J. Moser: $\alpha=1 / 6, \beta=5+\epsilon$, A. A. Karatsuba: $\alpha=5 / 32, \beta=2$, A. Ivić improved Karatsuba's exponent $5 / 32=0.15625$ to $0.1559458 \ldots$ A good reference for these results one can consult the book by A. Ivić ([3])

13. Let $\beta_{n}+i \gamma_{n}\left(\gamma_{n}>0\right)$ be all the zeros of $\zeta(s)$ arranged in the non-decreasing order of $\gamma_{n}$. Then E. C. Titchmarsh has proved that

$$
\gamma_{n+1}-\gamma_{n} \ll\left(\log \log \log \gamma_{n}\right)^{-1} .
$$

Myself and A. Sankaranarayanan have proved that either

$$
\gamma_{n+1}-\gamma_{n} \ll\left(\log \log \gamma_{n}\right)^{-1}
$$


or there is a zero in $\sigma \geq \frac{1}{2}+\left(\log \log \gamma_{n}\right)^{-1},\left|\gamma_{n}-t\right| \leq K \log \log \log t$ where $K>0$ is a constant and $\gamma_{n} \geq$ a large constant $c$.

14. Consider the theorem of E. Landau on the singularity of Dirichlet series with positive coefficients. R. Balasubramanian and myself have made it effective when the singularities are all poles. Try to make Landau's theorem effective in the most general case.

15. If $\sigma \geq 1-(\log t)^{-\alpha}(\alpha>0)$ is zero-free, it is not hard to prove that ([3])

$$
\pi(x)-\operatorname{li}(x)=O\left(x e^{-c Y}\right), Y=(\log x)^{\frac{1}{1+\alpha}}
$$

where $c>0$ is a constant. P. Turan has proved the converse by his method of power sums. His method is not simple. Try to give a simple proof.

Myself, A. Sankaranarayanan and K. Srinivas have proved (by a simple method) that things like

$$
\sum_{n \leq x} \mu(n)=O\left(x e^{-c Y}\right)
$$

and also

$$
\sum_{n_{1}, n_{2} \ldots n_{k} \leq x} \mu\left(n_{1}\right) \mu\left(n_{2}\right) \ldots \mu\left(n_{k}\right)=O\left(x e^{-c Y}\right)
$$

imply the corresponding zero-free region $([6])$. Here $k \geq 1$ is any fixed integer.

16. Vinogradov's theorem. Try to study the proof of the theorem

$$
|\pi(x)-\operatorname{li}(x)| \leq x\left\{\exp \left((\log x)^{\frac{3}{5}}(\log \log x)^{-\frac{1}{5}}\right)\right\}^{-\lambda}
$$

where $x \geq 1000$, and $\lambda>0$ is a certain absolute constant. This theorem is due to I. M. Vinogradov. He thought that he had a proof of

$$
|\pi(x)-\operatorname{li}(x)| \leq x\left\{\exp (\log x)^{\frac{3}{5}}\right\}^{-\lambda} .
$$

But there was a mistake. For the latest result interested reader may refer to [5]. I. M. Vinogradov's theorem is very deep and more than 80 years old. A trivial result is

$$
|\pi(x)-\operatorname{li}(x)| \leq x(\exp \sqrt{\log x})^{-\lambda}
$$

I mean that it does not require deep estimations.

17. These are not the only problems. You can frame it yourself or consult experts. 


\section{ReFERENCES}

[1] R. Balasubramanian, An improvement of a theorem of Titchmarsh on the mean square of $|\zeta(1 / 2+i t)|$. Proc. London Math. Soc., 36 (1978), 540-576.

[2] Hardy, G. H., Ramanujan: Twelve Lectures on Subjects Suggested by His Life and Work. 3rd ed. New York: Chelsea, 1999.

[3] A. Ivić, The Riemann zeta-function, The theory of the Riemann zeta-function with applications. A Wiley-Interscience Publication. John Wiley \& Sons, Inc., New York, (1985). xvi+517.

[4] R. Balasubramanian, Ramachandra, K., Transcendental numbers and a lemma in combinatorics. Proc. Sem. Combinatorics and Applications, Indian Stat. Inst., (1982), 5759

[5] K. Ramachandra and A. Sankaranarayanan., A remark on Vinogradov's mean-value theorem. J. Anal. 3 (1995), 111-129.

[6] K. Ramachandra, A. Sankaranarayanan and K.Srinivas., Notes on prime number theoremII. vol 72, Jr. Indian Math. Soc. 2005, no. 1-4, 13-18.

[7] K. Ramachandra and T. N. Shorey, On gaps between numbers with a large prime factor. Acta Arith 24 (1973), 99-111.

[8] M. Jutila., On numbers with a large prime factor. J. Indian Math. Soc. (N.S.) 38 (1974), $125-130]$

[9] Mihăilescu, P., Primary Cyclotomic Units and a Proof of Catalan's Conjecture. J. Reine angew. Math. 572 (2004), 167-195

[10] S. Ramanujan., Modular equations and approximations to $\pi$. Quart. J. Math. 45 (1914), 350-372, Collected papers of Srinivasa Ramanujan, 23-39, AMS Chelsea Publ., Providence, RI, 2000. 